\title{
Exploration of nonlocalities in ensembles consisting of bipartite quantum states
}

\author{
Ming-Yong Ye, ${ }^{1,2}$ Yan-Kui Bai, ${ }^{3}$ Xiu-Min Lin, ${ }^{2}$ and Z. D. Wang ${ }^{1}$ \\ ${ }^{1}$ Department of Physics and Center of Theoretical and Computational Physics, University of Hong Kong, Pokfulam Road, Hong Kong, \\ People's Republic of China \\ ${ }^{2}$ School of Physics and Optoelectronics Technology, Fujian Normal University, Fuzhou 350007, People's Republic of China \\ ${ }^{3}$ College of Physical Science and Information Engineering and Hebei Advance Thin Films Laboratory, Hebei Normal University, \\ Shijiazhuang, Hebei 050016, People's Republic of China \\ (Received 4 February 2009; published 25 January 2010)
}

\begin{abstract}
It is revealed that ensembles consisting of multipartite quantum states can exhibit different kinds of nonlocalities. An operational measure is introduced to quantify nonlocalities in ensembles consisting of bipartite quantum states. Various upper and lower bounds for the measure are estimated and the exact values for ensembles consisting of mutually orthogonal maximally entangled bipartite states are evaluated.
\end{abstract}

DOI: 10.1103/PhysRevA.81.014303

PACS number(s): 03.67.Mn, 03.65.Ud, 03.67.Hk

Introduction. Although mutually orthogonal multipartite quantum states are always distinguishable through joint measurements, it was found by Bennett et al. [1] in 1999 that there are ensembles of mutually orthogonal bipartite product states that cannot be distinguished by means of local operations and classical communication (LOCC); this phenomenon was referred to as the nonlocality without entanglement. The essence of this nonlocality is that the maximal information achievable through LOCC to distinguish an ensemble of mutually orthogonal multipartite states may be strictly less than that which could be obtained through joint measurements. Since the discovery of this phenomenon, substantial efforts have been devoted to searching for the conditions under which a given ensemble can exhibit such a kind of nonlocality [2-13]. As is known, any ensemble consisting of the four Bell states can exhibit the nonlocality [11], whereas the ensembles consisting of only two orthogonal states cannot [12]. As a latest result, it was indicated recently that the nonlocality exists in almost all ensembles consisting of more than $d$ mutually orthogonal $d^{\otimes n}$ states [13].

So far, most investigations on this intriguing ensemble nonlocality have been made on qualitative descriptions in analogy to quantum entanglement judgment (that is, finding conditions that can be used to check whether a given ensemble can exhibit the nonlocality), while the quantification of the nonlocality and its implications are still awaited. To our knowledge, quantification of the nonlocalities in ensembles has only been addressed in detail in Ref. [14], though how to quantify quantum entanglement has been intensively studied.

In this article we explore nonlocalities in ensembles consisting of bipartite quantum states and introduce an operational measure for them. The measure is defined through considering the tensor power $\varepsilon^{\otimes n}$, rather than the ensemble $\varepsilon$ itself, such that some results from information theory can be used directly. Our discussions focus mainly on ensembles whose states are mutually orthogonal, motivated by the following two questions: if an ensemble of mutually orthogonal bipartite states cannot be distinguished by LOCC, how much entanglement in addition to LOCC is needed [15]? and if they can be distinguished by LOCC, how much entanglement can be distilled in the process of locally distinguishing?

Entanglement charge. LOCC distinguishing the states in the ensemble $\varepsilon=\left\{p_{X}, \rho_{X}^{A B}\right\}$ can be conceived as a game.
Suppose that there is a classical information source producing symbol $X$ with probability $p_{X}$. If the source outputs symbol $X$, Alice and Bob will be given a quantum state $\rho_{X}^{A B}$. They know the ensemble $\varepsilon$ and their task is to determine the value of $X$ via a measurement implemented through LOCC. How much information they have gained about the value of $X$ can be described by the mutual information between $X$ and the measurement result $Y$,

$$
I(X ; Y)=H(X)+H(Y)-H(X Y),
$$

where $H(\cdot)$ is the Shannon entropy of the random variables. The value of $X$ can be determined through the measurement result $Y$ if and only if $I(X ; Y)=H(X)$ [16]. The maximal mutual information achievable through LOCC is called locally accessible information and it will be denoted by $I^{\mathrm{LOCC}}(\varepsilon)$. Similarly we can define $I^{\text {Global }}(\varepsilon)$, which is the maximal mutual information achievable through joint measurements and will be equal to $H(X)$ when the states are mutually orthogonal. Generally there is $I^{\mathrm{LOCC}}(\varepsilon) \leqslant I^{\mathrm{Global}}(\varepsilon) \leqslant H(X)$.

The tensor power of the ensemble $\varepsilon=\left\{p_{X}, \rho_{X}^{A B}\right\}$ is denoted as $\varepsilon^{\otimes n}=\left\{p_{X^{n}}, \rho_{X^{n}}^{A^{n} B^{n}}\right\}$, where $p_{X^{n}}=p_{X_{1}} p_{X_{2}}, \ldots, p_{X_{n}}$, $\rho_{X^{n}}^{A^{n} B^{n}}=\rho_{X_{1}}^{A_{1} B_{1}} \otimes \rho_{X_{2}}^{A_{2} B_{2}}, \ldots, \otimes \rho_{X_{n}}^{A_{n} B_{n}}$, and $X_{i}$ are independent and identically distributed classical variables as $X$. Now Alice holds $A^{n}$ and Bob holds $B^{n}$. To obtain the information about the value of $X^{n}$, they make a measurement that satisfies the following conditions: (1) the mutual information between $X^{n}$ and the measurement result $Y$ satisfies $I\left(X^{n} ; Y\right) \geqslant$ $I^{\text {Global }}\left(\varepsilon^{\otimes n}\right)-\delta_{n}$ with $\lim _{n \rightarrow \infty} \delta_{n}=0$; (2) it is implemented through LOCC plus $n \times \alpha_{n}$ ebits of entanglement; (3) when the measurement result $Y$ with the probability $p_{Y}$ is obtained, $n \times \beta_{n Y}$ ebits of entanglement is distilled. We now introduce a new quantity-entanglement charge, which is defined as

$$
N(\varepsilon)=\inf \lim _{n \rightarrow \infty}\left(\alpha_{n}-\sum_{Y} p_{Y} \times \beta_{n Y}\right),
$$

where the infimum operation is taken over all measurements satisfying the aforementioned conditions. $N(\varepsilon)$ is applicable to ensembles consisting of general bipartite states and its value can be positive, negative, and zero.

Two kinds of ensemble nonlocalities and their quantification. The ensembles with positive $N(\varepsilon)$ are different from those with negative $N(\varepsilon)$ in the sense that they can exhibit different 
kinds of nonlocalities. We focus on ensembles consisting of mutually orthogonal bipartite states hereafter. Now there is $I^{\text {Global }}\left(\varepsilon^{\otimes n}\right)=H\left(X^{n}\right)$ and the first condition required for the measurement can be interpreted that the states in the ensemble $\varepsilon$ will be distinguished with vanishing error.

In the case $N(\varepsilon)>0$, quantum entanglement is needed in addition to LOCC to distinguish the states in the ensemble $\varepsilon$ with vanishing error. The meaning of the positive entanglement charge $N(\varepsilon)$ can be manifested through the symbolic expression

$$
N(\varepsilon)[q q]+\mathrm{LOCC}_{\varepsilon}=>I^{\mathrm{Global}}(\varepsilon),
$$

where $[q q]$ means an ebit of quantum entanglement and $I^{\text {Global }}(\varepsilon)=H(X)$. Noting that $H(X)$ is the information needed to distinguish the states in $\varepsilon$, the positive $N(\varepsilon)$ quantifies the minimal nonlocal resources (entanglement) that are needed asymptotically in addition to LOCC to get the full information $H(X)$ to distinguish the states in $\varepsilon$. In this case we refer to the corresponding nonlocality exhibited by ensembles as information nonlocality and employ $N(\varepsilon)$ as a measure to quantify it.

In the case $N(\varepsilon)<0$, quantum entanglement is not needed in addition to LOCC to distinguish the states in the ensemble $\varepsilon$ with vanishing error (if the entanglement is still needed to assist the process, it could be viewed as a kind of catalyst), and additionally some entanglement can be distilled. Similarly the meaning of the negative entanglement charge $N(\varepsilon)$ can be manifested through the expression

$$
\operatorname{LOCC}_{\varepsilon}=>I^{\text {Global }}(\varepsilon)+|N(\varepsilon)|[q q] .
$$

In this case, the ensemble $\varepsilon$ has no information nonlocality; however, it still exhibits some kind of nonlocality since certain entanglement can be distilled. Hereafter we may refer to such kind of ensemble nonlocality as entanglement nonlocality and employ $|N(\varepsilon)|$ as its measure since it quantifies the maximal quantum entanglement that can be distilled in the asymptotic limit.

Interestingly, in the case $N(\varepsilon)=0$, the ensemble $\epsilon$ has neither the information nonlocality nor the entanglement nonlocality mentioned previously. As a typical example, $N(\varepsilon)=0$ for ensembles that consist of LOCC distinguishable product states [17].

Bounds for entanglement charge. Although the entanglement charge $N(\varepsilon)$ is usually hard to compute, some useful bounds for it can be obtained. Bennett et al. considered how much quantum transmission is needed to complete a special distinguishing measurement in Ref. [1], where an upper bound for our defined $N(\varepsilon)$ is implied, that is, $N(\varepsilon) \leqslant S\left(\rho^{A}\right)$, where $\rho^{A}=\operatorname{Tr}_{B}\left(\sum_{X} p_{X} \rho_{X}^{A B}\right)$ and $S(\cdot)$ is the quantum entropy. This can be obtained through the protocol that Alice first compresses her state [18] and teleports it to Bob [19] and Bob then distinguishes the states locally. The following are more tight upper bounds.

Theorem 1. Suppose $\varepsilon=\left\{p_{X}, \rho_{X}^{A B}\right\}$ is an ensemble consisting of mutually orthogonal bipartite states. The entanglement charge will satisfy

$$
\begin{aligned}
& N(\varepsilon) \leqslant S(A \mid B)=S\left(\rho^{A B}\right)-S\left(\rho^{B}\right), \\
& N(\varepsilon) \leqslant S(B \mid A)=S\left(\rho^{A B}\right)-S\left(\rho^{A}\right),
\end{aligned}
$$

where $\rho^{A B}=\sum_{X} p_{X} \rho_{X}^{A B}, \rho^{B}=\operatorname{Tr}_{A} \rho^{A B}, \rho^{A}=\operatorname{Tr}_{B} \rho^{A B}$, and $S(\cdot)$ is the quantum entropy.

Proof. The theorem can be derived from the quantum state merging [20,21]. To distinguish the states in the ensemble $\varepsilon^{\otimes n}$, the part $A^{n}$ on Alice's side can be merged to Bob and then he distinguishes the states locally. In the process of merging [20,21], the net consumed entanglement can be $S(A \mid B)$ ebits, so Eq. (5) is obtained. If the part $B^{n}$ on Bob's side is first merged to Alice and then she distinguishes the states locally, similarly the net consumed entanglement can be $S(B \mid A)$ ebits, so Eq. (6) is obtained.

The above upper bounds depend only on the state $\rho^{A B}$, so different ensembles may have the same upper bound. It is possible to get a smaller bound if we examine the states in the ensemble carefully since it is possible that only a part of $A$ needs to be merged to Bob and then the states become LOCC distinguishable.

Theorem 2. Suppose $\varepsilon=\left\{p_{X}, \rho_{X}^{A B}\right\}$ is an ensemble consisting of mutually orthogonal bipartite pure states. The entanglement charge will satisfy

$$
N(\varepsilon) \geqslant \sum p_{X} S\left(\rho_{X}^{A}\right)-I_{\rho^{A B}}(A ; B)
$$

where $S(\cdot)$ is the quantum entropy and $I_{\rho^{A B}}(A ; B)=S\left(\rho^{A}\right)+$ $S\left(\rho^{B}\right)-S\left(\rho^{A B}\right)$ is the quantum mutual information with $\rho^{A B}=\sum_{X} p_{X} \rho_{X}^{A B}, \rho^{A}=\operatorname{Tr}_{B} \rho^{A B}, \rho^{B}=\operatorname{Tr}_{A} \rho^{A B}$, and $\rho_{X}^{A}=$ $\operatorname{Tr}_{B} \rho_{X}^{A B}$.

The quantum mutual information $I_{\rho^{A B}}(A ; B)$ is always nonnegative and it can be regarded as a quantification of the total correlation between $A$ and $B$, so Eq. (7) means that the entanglement charge is not smaller than the average entanglement of the states in the ensemble minus the total correlation between $A$ and $B$. When $\rho^{A B}=\rho^{A} \otimes \rho^{B}$ there is $I_{\rho^{A B}}(A ; B)=0$ and $N(\varepsilon) \geqslant \sum p_{X} S\left(\rho_{X}^{A}\right)$; the average entanglement of the states in the ensemble is a lower bound of the entanglement charge. This case will happen when we consider an ensemble consisting of full basis states with equal probability.

Proof. According to the definition of $N(\varepsilon)$ we should consider distinguishing the states of the ensemble $\varepsilon^{\otimes n}=$ $\left\{p_{X^{n}}, \rho_{X^{n}}^{A^{n} B^{n}}\right\}$ using LOCC plus $n \times \alpha_{n}$ ebits of entanglement. It is equivalent to distinguish the states of the ensemble $\left\{p_{X^{n}}, \rho_{X^{n}}^{A^{n} B^{n}} \otimes \Phi_{n}^{A_{0} B_{0}}\right\}$ using LOCC only, where $\Phi_{n}^{A_{0} B_{0}}$ is a bipartite pure state with $S\left(\Phi_{n}^{A_{0}}\right)=S\left(\Phi_{n}^{B_{0}}\right)=n \times \alpha_{n}$. The mutual information between $X^{n}$ and the measurement result $Y$ will satisfy $[22,23]$

$$
\begin{aligned}
I\left(X^{n} ; Y\right) \leqslant & n\left[S\left(\rho^{B}\right)+S\left(\rho^{A}\right)-\sum p_{X} S\left(\rho_{X}^{A}\right)\right] \\
& +n\left(\alpha_{n}-\sum p_{Y} \beta_{n Y}\right) .
\end{aligned}
$$

Noting that $I\left(X^{n} ; Y\right) \geqslant I^{\text {Global }}\left(\varepsilon^{\otimes n}\right)-\delta_{n}$ is required and there is $I^{\text {Global }}\left(\varepsilon^{\otimes n}\right)=n S\left(\rho^{A B}\right)$, we can get

$$
\alpha_{n}-\sum p_{Y} \beta_{n Y} \geqslant \sum p_{X} S\left(\rho_{X}^{A}\right)-I_{\rho^{A B}}(A ; B)-\delta_{n} / n \text {. }
$$

Since $\delta_{n} / n$ will go to zero in the asymptotic limit, we can get Eq. (7) from Eq. (9).

The above two theorems give upper and lower bounds on $N(\varepsilon)$. It is valuable to know when the upper and the 
lower bounds will be close. We first rewrite the lower bound expression in Theorem 2 as

$$
N(\varepsilon) \geqslant S(A \mid B)-\chi^{A}(\varepsilon)=S(B \mid A)-\chi^{B}(\varepsilon),
$$

where $\chi^{A}(\varepsilon)=S\left(\rho^{A}\right)-\sum p_{X} S\left(\rho_{X}^{A}\right)$ and $\chi^{B}(\varepsilon)=S\left(\rho^{B}\right)-$ $\sum p_{X} S\left(\rho_{X}^{B}\right)$ are the Holevo information of the ensembles seen by Alice and Bob, respectively. As for ensembles consisting of orthogonal pure states, it is not hard to find that the contents of the two theorems can be summarized as

$$
S(A \mid B)-\chi^{A}(\varepsilon) \leqslant N(\varepsilon) \leqslant S(A \mid B),
$$

when $\chi^{A}(\varepsilon) \leqslant \chi^{B}(\varepsilon)$, or

$$
S(B \mid A)-\chi^{B}(\varepsilon) \leqslant N(\varepsilon) \leqslant S(B \mid A),
$$

when $\chi^{B}(\varepsilon) \leqslant \chi^{A}(\varepsilon)$. From Eqs. (11) and (12), we know that the upper and the lower bounds will be closer if ever $\chi^{A}(\varepsilon)$ or $\chi^{B}(\varepsilon)$ is smaller. Noting that $\chi^{A}(\varepsilon)$ is the upper bound of the information about the value of $X$ that Alice can gain solely [16], Eq. (11) means that the difference between the bounds will be small if Alice can gain little information about the value of $X$ without cooperation with Bob. When $\chi^{A}(\varepsilon)=0$ or $\chi^{B}(\varepsilon)=0$, the exact value of $N(\varepsilon)$ can be obtained. This occurs only when all $\rho_{X}^{A}$ (or $\rho_{X}^{B}$ ) are the same. Consequently, we have the following result.

Corollary 1. Suppose that $\varepsilon=\left\{p_{X}, \rho_{X}^{A B}\right\}$ is an ensemble consisting of mutually orthogonal $d \times d$ maximally entangled pure states. The entanglement charge will be

$$
N(\varepsilon)=S\left(\rho^{A B}\right)-S\left(\rho^{B}\right)=H(X)-\log d,
$$

where $H(\cdot)$ is the Shannon entropy, $\rho^{A B}=\sum_{X} p_{X} \rho_{X}^{A B}$, and $\rho^{B}=\operatorname{Tr}_{A} \rho^{A B}$.

The corollary is true since all $\rho_{X}^{A}=\operatorname{Tr}_{B} \rho_{X}^{A B}$ and $\rho_{X}^{B}=$ $\operatorname{Tr}_{A} \rho_{X}^{A B}$ are the same maximally mixed state, so there are both $\chi^{A}(\varepsilon)=0$ and $\chi^{B}(\varepsilon)=0$; the upper and the lower bounds of $N(\varepsilon)$ become the same value. As is known, the four Bell states cannot be distinguished by LOCC [11]; however, the corollary indicates that the entanglement charge $N(\varepsilon)$ of an ensemble consisting of these four states can be any value between -1 and 1 dependent on their probabilities, so it may have the information nonlocality, the entanglement nonlocality, or neither. The reason is that whether the states in the ensemble $\varepsilon$ are LOCC indistinguishable depends only on its states, while whether the ensemble has the information nonlocality or the entanglement nonlocality depends not only on its states but also on the probabilities of the states.

There are other ways to obtain upper bounds for $N(\varepsilon)$. For any ensemble $\varepsilon=\left\{p_{X}, \rho_{X}^{A B}\right\}$ whose states are mutually orthogonal, there exist nonlocal unitary operations $U^{A B}$ such that the states of the ensemble $\bar{\varepsilon}=\left\{p_{X}, U^{A B} \rho_{X}^{A B} U^{\dagger A B}\right\}$ are LOCC distinguishable. For an example we consider the ensemble $\varepsilon$ consisting of the following states:

$$
\begin{array}{ll}
U(-\theta)|0\rangle_{A}|0\rangle_{B}, & U(-\theta)|0\rangle_{A}|1\rangle_{B}, \\
U(-\theta)|1\rangle_{A}|0\rangle_{B}, & U(-\theta)|1\rangle_{A}|1\rangle_{B},
\end{array}
$$

where $U(-\theta)=\exp \left\{-i \theta \sigma_{x}^{A} \sigma_{x}^{B}\right\}$. A similar example appears in Ref. [24] where the nonlocal measurements are discussed.
The states have the same entanglement,

$$
H\left(\cos ^{2} \theta\right)=-\cos ^{2} \theta \log _{2} \cos ^{2} \theta-\sin ^{2} \theta \log _{2} \sin ^{2} \theta .
$$

We first consider the case where the states have equal probability. In this case the upper bounds obtained from Theorem 1 can be expressed as $N(\varepsilon) \leqslant 1$, which is not satisfactory. Note that if Alice and Bob implement $U(\theta)$ on $A B$ the changed states will be LOCC distinguishable. The operation $U(\theta)$ can be implemented through LOCC plus some entanglement. The average entanglement $\bar{E}(\theta)$ needed to implement $U(\theta)$ is an upper bound of the entanglement charge $N(\varepsilon)$, that is, $N(\varepsilon) \leqslant \bar{E}(\theta)$. Several expressions for $\bar{E}(\theta)$ are given [24-27], and the one given in Ref. [25] shows that $\bar{E}(\theta)$ will be smaller than unit when $2 \theta \leqslant 0.75$. It means when $2 \theta \leqslant 0.75$, the upper bound expression for $N(\varepsilon)$ obtained by calculating the average entanglement to implement $U(\theta)$ will be better than that in Theorem 1. However, this may not be true when the states have different probabilities $p_{X}$. To see this we note that $S\left(\rho^{A}\right) \geqslant \sum_{X} p_{X} S\left(\rho_{X}^{A}\right)=H\left(\cos ^{2} \theta\right)$, so from Theorem 1 there is

$$
N(\varepsilon) \leqslant S\left(\rho^{A B}\right)-S\left(\rho^{A}\right) \leqslant H(X)-H\left(\cos ^{2} \theta\right) .
$$

The above upper bound for $N(\varepsilon)$ depends on the probabilities $p_{X}$ and surely it will be smaller than $\bar{E}(\theta)$ when $H(X)$ is smaller than $\bar{E}(\theta)+H\left(\cos ^{2} \theta\right)$.

Discussion. Ensembles consisting of mutually orthogonal bipartite states can be classified into three categories according to the value of the entanglement charge $N(\varepsilon)$ : one has the information nonlocality, one has the entanglement nonlocality, and the third has neither. For an ensemble, if it has the information nonlocality obviously the states in it are LOCC indistinguishable; however, the inverse may not be true. The reason is that the value of the entanglement charge $N(\varepsilon)$ depends not only on the states in the ensemble $\varepsilon$ but also on the probabilities of the states, whereas whether the states in the ensemble $\varepsilon$ are LOCC indistinguishable does not depend on their probabilities.

The concepts of information nonlocality and entanglement nonlocality can be extended to ensembles consisting of general bipartite quantum states since the definition of the entanglement charge $N(\varepsilon)$ does not depend on the orthogonality of the states. The extension is straightforward and also the entanglement charge $N(\varepsilon)$ can be used as a measure for them. The upper bound expressions in Theorem 1 are also applicable for general ensembles but the lower bound expression in Theorem 2 needs to be changed into

$$
N(\varepsilon) \geqslant \sum p_{X} S\left(\rho_{X}^{A}\right)-I_{\rho^{A B}}(A ; B)-\Delta(\varepsilon),
$$

where $\Delta(\varepsilon)=S\left(\rho^{A B}\right)-I^{\text {Global }}(\varepsilon)$, which can be obtained in the same way as the states are mutually orthogonal.

When the states in the ensemble $\varepsilon=\left\{p_{X}, \rho_{X}^{A B}\right\}$ are not mutually orthogonal, they cannot be distinguished even through joint measurements, and the mutual information $I^{\text {Global }}(\varepsilon)$ is the maximal information about the value of $X$ that can be achieved through physical measurements. Nevertheless, the entanglement charge $N(\varepsilon)$ still has its operational meaning. The positive $N(\varepsilon)$ just quantifies the minimal entanglement that is needed asymptotically in addition to LOCC to achieve 
$I^{\text {Global }}(\varepsilon)$. When $N(\varepsilon)$ is negative, asymptotically LOCC can get the information $I^{\text {Global }}(\varepsilon)$, and additionally at most $|N(\varepsilon)|$ ebits of entanglement can be distilled.

Conclusion. We have introduced the entanglement charge as a measure to quantify nonlocalities in ensembles consisting of bipartite quantum states. We have estimated various upper and lower bounds for the entanglement charge and evaluated the exact values for ensembles consisting of mutually orthogonal maximally entangled bipartite pure states. The present work is expected to evoke more profound understandings of nonlocalities in ensembles.

This work was supported by the RGC of Hong Kong under HKU7051/06P and HKU7044/08P, the fund of Hebei Normal University, the Foundation for Universities in Fujian Province (No. 2007F5041), NSF-China (Nos. 60878059, 10947147 and 10905016), and the National Basic Research Program of China (No. 2006CB921800).
[1] C. H. Bennett, D. P. DiVincenzo, C. A. Fuchs, T. Mor, E. Rains, P. W. Shor, J. A. Smolin, and W. K. Wootters, Phys. Rev. A 59, 1070 (1999).

[2] M. Y. Ye, W. Jiang, P. X. Chen, Y. S. Zhang, Z. W. Zhou, and G. C. Guo, Phys. Rev. A 76, 032329 (2007).

[3] C. H. Bennett, D. P. DiVincenzo, T. Mor, P. W. Shor, J. A. Smolin, and B. M. Terhal, Phys. Rev. Lett. 82, 5385 (1999).

[4] M. Horodecki, A. Sen(De), U. Sen, and K. Horodecki, Phys. Rev. Lett. 90, 047902 (2003).

[5] P. X. Chen and C. Z. Li, Phys. Rev. A 68, 062107 (2003).

[6] J. Watrous, Phys. Rev. Lett. 95, 080505 (2005).

[7] H. Fan, Phys. Rev. Lett. 92, 177905 (2004).

[8] M. Hayashi, D. Markham, M. Murao, M. Owari, and S. Virmani, Phys. Rev. Lett. 96, 040501 (2006).

[9] Y. Xin and R. Y. Duan, Phys. Rev. A 77, 012315 (2008).

[10] J. Walgate and L. Hardy, Phys. Rev. Lett. 89, 147901 (2002).

[11] S. Ghosh, G. Kar, A. Roy, A. Sen(De), and U. Sen, Phys. Rev. Lett. 87, 277902 (2001).

[12] J. Walgate, A. J. Short, L. Hardy, and V. Vedral, Phys. Rev. Lett. 85, 4972 (2000).

[13] S. M. Cohen, Phys. Rev. A 77, 060309 (R) (2008).

[14] M. Horodecki, A. Sen(De), and U. Sen, Phys. Rev. A 75, 062329 (2007).

[15] S. M. Cohen, Phys. Rev. A 77, 012304 (2008).
[16] M. A. Nielsen and I. L. Chuang, Quantum Computation and Quantum Information (Cambridge University Press, Cambridge, UK, 2000).

[17] Notably, a single quantum state may also be regarded as a special ensemble, and $N(\varepsilon)=-D(\varepsilon)$ in this case, where $D(\varepsilon)$ denotes the distillable entanglement of the state.

[18] B. Schumacher, Phys. Rev. A 51, 2738 (1995).

[19] C. H. Bennett, G. Brassard, C. Crépeau, R. Jozsa, A. Peres, and W. K. Wootters, Phys. Rev. Lett. 70, 1895 (1993).

[20] M. Horodecki, J. Oppenheim, and A. Winter, Nature (London), 436, 673 (2005).

[21] M. Horodecki, J. Oppenheim, and A. Winter, Commun. Math. Phys. 269, 107 (2007).

[22] P. Badziag, M. Horodecki, A. Sen(De), and U. Sen, Phys. Rev. Lett. 91, 117901 (2003).

[23] M. Horodecki, J. Oppenheim, A. Sen(De), and U. Sen, Phys. Rev. Lett. 93, 170503 (2004).

[24] S. Bandyopadhyay, G. Brassard, S. Kimmel, and W. K. Wootters, Phys. Rev. A 80, 012313 (2009).

[25] M. Y. Ye, Y. S. Zhang, and G. C. Guo, Phys. Rev. A 73, 032337 (2006).

[26] J. I. Cirac, W. Dür, B. Kraus, and M. Lewenstein, Phys. Rev. Lett. 86, 544 (2001).

[27] D. W. Berry, Phys. Rev. A 75, 032349 (2007). 